\title{
THE FIRST BETTI NUMBER OF A COMPACT ALMOST TACHIBANA SPACE
}

\author{
SEIICHI YAMAGUCHI
}

\section{Introduction}

It is well known that the $p$-th Betti number of a compact Kählerian space is zero or even if $p$ is odd [2]. A similar result is known for a compact Sasakian space [1], [6], [7]. In particular, the first Betti number is zero or even in a compact Sasakian space.

The purpose of this paper is to give the analogy for the first Betti number of a compact Tachibana space (=nearly Kähler space [3], $=K$-space [4]).

\section{Preliminaries}

Let $M$ be an $n$-dimensional almost Hermitian space with positive definite metric $g=\left(g_{j i}\right)$ and almost complex structure $J=\left(J_{i}{ }^{j}\right),(i, j, \cdots=1, \cdots, n)$.

A 1 -form $u$ in $M$ is called a covariant almost analytic form [4] if it satisfies the equation

$$
\nabla_{j}\left(J_{i}^{r} u_{r}\right)=u_{r} \nabla_{i} J_{j}^{r}-J_{j}^{r} \nabla_{r} u_{i}
$$

or equivalently

$$
\nabla_{j}\left(J_{i}{ }^{r} u_{r}\right)-\nabla_{i}\left(J_{j}{ }^{r} u_{r}\right)=J_{j}^{r}\left(\nabla_{r} u_{i}-\nabla_{i} u_{r}\right),
$$

where $V$ denotes the operator of covariant derivative with respect to the Riemannian connection.

An almost Hermitian space is called an almost Tachibana space (resp. a Kählerian space) if the associated 2-form $\bar{J}=\frac{1}{2} J_{j i} d x^{j} \wedge d x^{i}$ is a Killing 2form (resp. parallel), where we put $J_{j i}=g_{i r} J_{j}{ }^{r}$ and $\left\{x^{i}\right\}$ is a local coordinate system of $M$.

Then the following theorems are known:

Theorem A [9]. A necessary and sufficient condition for a 1-form $u$ in a compact Kählerian space to be covariant analytic is that the 1-form $u$ be harmonic.

Theorem B [4]. In a compact almost Tachibana space, a necessary and sufficient condition for a 1-form $u=\left(u_{i}\right)$ to be covariant almost analytic is that $u$ and $\bar{u}=\left(J_{i}{ }^{r} u_{r}\right)$ both be harmonic.

Received September 8, 1972, and, in revised form, April 3, 1973. 
Throughout this paper, we shall deal with an almost Tachibana space $M$, that is, an almost Hermitian space satisfying

$$
\nabla_{j} J_{i h}+\nabla_{i} J_{j h}=0 \text {. }
$$

We shall recall the identities in $M$, which are necessary for later use.

The following relations are well known [4], [8], [9]:

$$
\begin{gathered}
J_{j}{ }^{r} R_{r i}+J_{i}{ }^{r} R_{r j}=0, \\
\nabla^{r} \nabla_{r} J_{j i}=R_{j r} J_{i}{ }^{r}-\frac{1}{2} J^{r s} R_{r s j i} .
\end{gathered}
$$

Next, let $u$ be any 1 -form. Then by virtue of the Ricci's identity we can obtain

$$
J^{r s} \nabla_{r} \nabla_{s} u_{i}=-\frac{1}{2} J^{r s} R_{r s i}{ }^{t} u_{t} .
$$

If $u$ is a harmonic 1 -form, then we have

$$
\nabla_{j} u_{i}-\nabla_{i} u_{j}=0, \quad \nabla^{r} \nabla_{r} u_{i}-R_{i}{ }^{r} u_{r}=0,
$$

which are valid in any Riemannian space.

\section{Theorems}

Let us prove the following theorem.

Theorem 2.1. In a compact almost Tachibana space $M$, if $u$ is a harmonic 1-form, then $\bar{u}=\left(J_{i}{ }^{r} u_{r}\right)$ is also so.

Proof. Since $u$ is a harmonic 1 -form, we have

$$
\nabla_{i}\left(J_{j}{ }^{r} u_{r}\right)-\nabla_{j}\left(J_{i}^{r} u_{r}\right)=2 u_{r} \nabla_{i} J_{j}^{r}+J_{j}^{r} \nabla_{r} u_{i}-J_{i}^{r} \nabla_{r} u_{j},
$$

and therefore

$$
\begin{aligned}
& \left(u_{r} \nabla_{i} J_{j}{ }^{r}\right) u_{s} \nabla^{i} J^{j s}+\frac{1}{2}\left(J_{i}{ }^{r} \nabla_{r} u_{j}-J_{j}{ }^{r} \nabla_{r} u_{i}\right)\left(J^{i s} \nabla_{s} u^{j}-J^{j s} \nabla_{s} u^{i}\right) \\
& =\left(u_{r} \nabla_{i} J_{j}{ }^{r}\right) u_{s} \nabla^{i} J^{j s}+\left(J_{i}{ }^{r} \nabla_{r} u_{j}\right) J^{j s} \nabla_{s} u^{j}-\left(J_{j}^{r} \nabla_{r} u_{i}\right) J^{i s} \nabla_{s} u^{j} \\
& =\left(u_{r} \nabla_{i} J_{j}^{r}\right) \nabla^{i}\left(J^{j s} u_{s}\right)-\left(u_{r} \nabla_{i} J_{j}{ }^{r}\right) J^{j s} \nabla^{i} u_{s} \\
& +\left(J_{i}{ }^{r} \nabla_{r} u_{j}\right) J^{i s} \nabla_{s} u^{j}-\left(J_{j}{ }^{r} \nabla_{r} u_{i}\right) J^{i s} \nabla_{s} u^{j} \\
& =\left(u_{s} \nabla_{i} J_{j}^{s}\right) \nabla^{i}\left(J^{j r} u_{r}\right)+\left(J_{j}^{s} \nabla_{i} u_{s}\right) \nabla^{i}\left(J^{j r} u_{r}\right) \\
& -\left(J_{i}{ }^{s} \nabla_{s} u_{j}\right) \nabla^{i}\left(J^{j r} u_{r}\right)+3\left(u_{r} \nabla_{j} J_{i}^{r}\right) J^{j s} \nabla_{s} u^{i} \\
& =\left(\nabla^{i}\left(J^{j r} u_{r}\right)\right)\left[\nabla_{i}\left(J_{j}{ }^{s} u_{s}\right)-J_{i}^{s} \nabla_{j} u_{s}\right]+3\left(u_{r} \nabla_{j} J_{i}^{r}\right) J^{j r} \nabla_{r} u^{i} \\
& =-\left(J^{j s} u_{s}\right) \nabla^{i} \nabla_{i}\left(J_{j}^{r} u_{r}\right)+\left({ }^{j s} u_{s}\right) \nabla^{i}\left(J_{i}{ }^{r} \nabla_{j} u_{r}\right)+3\left(u_{r} \nabla_{j} J_{i}^{r}\right) J^{j s} \nabla_{s} u^{i} \\
& +\frac{1}{2} \nabla^{i} \nabla_{i}\left(J^{j s} u_{s} J_{j}^{r} u_{r}\right)-\nabla^{i}\left(J^{j s} u_{s} J_{i}{ }^{r} \nabla_{j} u_{r}\right) \text {. }
\end{aligned}
$$

On the other hand, making use of $(1.1), \cdots,(1.5)$ we easily see that 


$$
\nabla^{r}\left(J_{r}^{s} \nabla_{s} u_{i}\right)=\nabla^{r} \nabla_{r}\left(J_{i}^{s} u_{s}\right), \quad\left(u_{r} \nabla_{i} J_{j}^{r}\right) J^{i s} \nabla_{s} u^{j}=0 .
$$

Hence, by Green's theorem and the obvious fact that $\nabla^{r}\left(J_{r}{ }^{s} u_{s}\right)=0$, the theorem is proved.

As a corollary of this theorem, we obtain

Theorem 2.2. The first Betti number of a compact almost Tachibana space is zero or even.

By virtue of Theorem $B$ and Theorem 2.1, we get

Theorem 2.3. In a compact almost Tachibana space, a necessary and sufficient condition for a 1-form $u$ to be covariant almost analytic is that $u$ be harmonic.

The author would like to express his hearty thanks to Professors S. Tachibana and Y. Ogawa for their criticisms and advices, and also to the referee for his suggestions regarding the revision of this paper.

\section{References}

[1] T. Fujitani, Complex-valued differential forms on normal contact Riemannian manifolds, Tôhoku Math. J. 18 (1966) 349-361.

[2] S. I. Goldberg, Curvature and homology, Academic Press, New York, 1962.

[ 3 ] A. Gray, Nearly Kähler manifolds, J. Differential Geometry 4 (1970) 283-309.

[4] S. Tachibana, On almost-analytic vector in certain almost Hermitian manifolds, Tôhoku Math. J. 11 (1959) 351-363.

[ 5 ] — Analytic tensor and its generalization, Tôhoku Math. J. 12 (1960) 208-221.

[6] - On harmonic tensors in compact Sasakian spaces, Tôhoku Math. J. 17 (1965) 271-284.

[7] S. Tachibana \& Y. Ogawa, On the second Betti number of a compact Sasakian space, Natur. Sci. Rep. Ochanomizu Univ. 17 (1966) 27-32.

[ 8 ] S. Yamaguchi, G. Chüman \& M. Matsumoto, On a special almost Tachibana space, Tensor, N. S. 24 (1972) 351-354.

[9] K. Yano, Differential geometry on complex and almost complex spaces, Pergamon Press, Oxford, 1965.

SCIENCE University of ToKyo 
